\title{
A beautiful prize
}

\author{
The 2011 Nobel Prize in Chemistry awarded for the discovery of quasicrystals recognizes a breakthrough \\ that has fundamental scientific consequences.
}

Dan Shechtman's reaction after seeing the seemingly impossible electron diffraction pattern emerging from an alloy of aluminium and manganese was one of surprise and scepticism. It was only after excluding, through further experiments, the possible artefacts that could have produced such a result that he convinced himself that the ten-fold symmetry pattern he had observed corresponded to a compound with rotational but not translational symmetry ${ }^{1}$, hence ordered but aperiodic.

It was April 1982, and the belief that crystals must be periodic was so strong at the time that Shechtman's results were met with outrage and criticism, even after publication, and not least by an authority such as Linus Pauling ${ }^{2}$. It was only Shechtman's perseverance in challenging the established knowledge, even at the risk of derision and isolation, that would eventually lead to wide recognition, and finally to the award of the 2011 Nobel Prize in Chemistry.

A great part of the fascination for quasicrystals - so defined by Levine and Steinhardt in a paper published only weeks after Shechtman's ${ }^{3}$ - is owed to their unusual and mesmerizing, though mathematically well defined, atomic structure. A structure that, as shown by Lu and Steinhardt ${ }^{4}$ has similarities with the decoration patterns using girih tiles made by Islamic artists several centuries ago. Some of these patterns can still be admired, for example, in the Mausoleum of Oljeitu in Soltaniyeh in Iran (pictured); in the case of the Darb-i Imam shrine (also in Iran) they resemble a proper quasicrystalline structure in two dimensions. From a theoretical perspective, the possibility of tiling up a two-dimensional space with a five- or ten-fold rotational symmetry had been challenging mathematicians for a long time, until it was elegantly solved by Roger Penrose in the $1970 \mathrm{~s}^{5}$. These studies proved essential in determining the type of arrangements that atoms assume in a quasicrystal, although it is fair to say that the possibility that such patterns could occur in real crystals as energetically favoured phases was initially surprising.

The aperiodic structure of quasicrystalline materials has enormous consequences for their electronic, thermal and mechanical properties. Most quasicrystals are composed of metallic elements, but the electrical

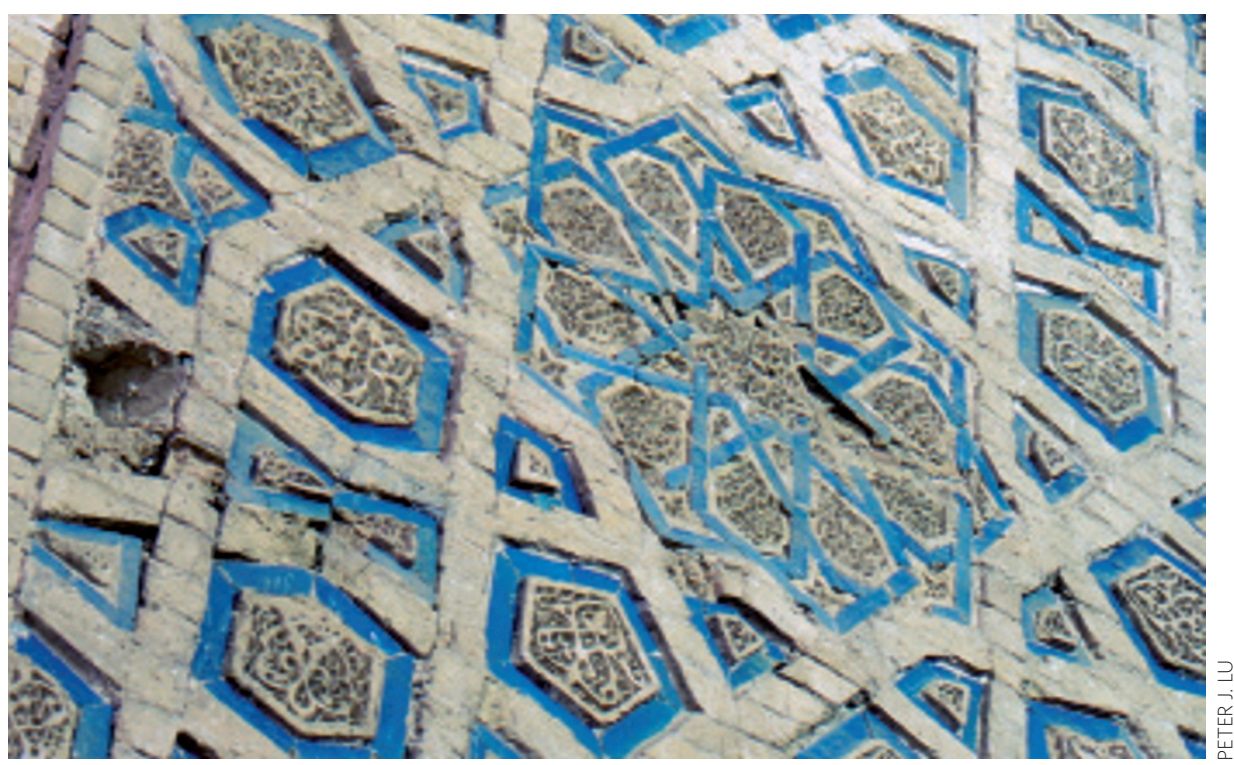

conductivity is not metallic at all, and increases with temperature in a similar fashion to that of semiconducting crystals. The aperiodicity also leads to very low thermal conductivity, as well as unique hardness and low friction.

Undoubtedly, the interest in quasicrystals in the past three decades has also been fuelled by the prospects of technological applications. Low friction could be applied to microand nanomechanical systems whereas low thermal conductivity could lead to efficient thermoelectric devices and thermal barriers. Other applications such as light absorption and sensing, catalysis, hydrogen storage and even cookware have been pursued ${ }^{6}$.

It is fair to say, however, that despite some isolated examples - like the production of non-stick pans that have a quasicrystalline coating - quasicrystals have hardly changed our day-to-day life. Whether it is appropriate to award the Nobel Prize to a discovery with potential technological applications before such applications are successfully realized is debatable. But it is hard to disagree with the fact that the discovery of materials that should not exist, that have provided a completely new perspective on a whole subject area, and that have raised the interest and excitement of a wide range of multidisciplinary scientists, deserves such important recognition.

This is precisely the case for quasicrystals. More widespread applications may come.
But as elegantly argued by the quasicrystals expert Jean-Marie Dubois in his book Useful Quasicrystals, the value of these materials is not necessarily to be sought in their relevance for technology ${ }^{5}$; instead, we should consider that they "have revolutionized our description of solid matter". In light of the discovery, the definition of crystal had to be officially changed from a periodic structure to one that produces a discrete diffraction pattern. Quasicrystals also provide a unique playground for scientists of different backgrounds to synthesize new compounds and to understand their very unusual properties.

At times when too much emphasis is put on technological relevance of scientific work at the expense of scientific curiosity, the Nobel Prize for a discovery that has had a primarily fundamental scientific impact is most welcomed. Thus, our most sincere congratulations go to Dan Shechtman, in recognition of his discovery and of his struggle to affirm his ideas against the scientific establishment.

\footnotetext{
References

1. Shechtman, D., Blech, I., Gratias, D., Cahn, J. W., Phys. Rev. Lett. 53, 1951-1954 (1984)

2. Pauling, L., Nature 317, 512-514 (1985).

3. Levine, D., Steinhardt, P. J., Phys Rev. Lett. 53, 2477-2480 (1984)

4. Lu, P. J., Steinhardt, P. J., Science 315, 1106-1110 (2007).

5. Penrose, R., Bull. Inst. Math. Applic. 10, 266-271 (1974).

6. Dubois, J. M., Useful Quasicrystals (World Scientific, 2005).
} 